\title{
X-ray Properties of Spitzer/IRAC Selected AGNs
}

\author{
Tuba İkiz ${ }^{1,2}$ (D) \\ ${ }^{1}$ Department of Astronomy and Space Sciences, Faculty of Science, Atatürk University, Erzurum, Turkey \\ ${ }^{2}$ Atatürk University Astrophysics Research and Application Center (ATASAM), Erzurum, Turkey
}

Geliş / Received: 30/05/2021, Kabul / Accepted: 04/07/2021

\begin{abstract}
Spitzer/IRAC color selection is a powerful tool to identify hot accreting nuclei, that is to say AGN, in galaxies. In this study, mid-infrared detected candidate 36 AGNs are used that are selected from the Spitzer Survey of Stellar Structures in Galaxies $\left(S^{4} \mathrm{G}\right)$ sample consisting of more than 2500 galaxies together with its extension sample of more than 400 galaxies by Ikiz et al., (2020). Mid-infrared color selection method is tested by examining the X-ray properties of the galaxies via the XMM-Newton and Chandra. Using the X-ray data, we demonstrate that galaxies displaying hot mid-infrared nuclei stand out as (candidate) active galaxies. $64 \%$ of mid-infrared-selected AGN are detected at X-ray energies in XMM-Newton and Chandra data. It has been hypothesized that IRAC sources with AGN colors that lack X-ray detections are predominantly highluminosity AGN that are obscure.
\end{abstract}

Key words: galaxies: active - galaxies: nuclei - infrared: galaxies - X-rays: galaxies

\section{Spitzer/IRAC Seçilmiş AGN'lerin X-ışın Özellikleri}

\section{$\ddot{\mathbf{O z}}$}

Spitzer/IRAC renk seçimi, galaksilerdeki sıcak madde biriktiren çekirdekleri, bir başka ifadeyle AGN'i tanımlamak için güçlü bir araçtır. Bu çalışmada İkiz et al., (2020) tarafından 2500'den fazla galaksiden oluşan Galaksilerdeki Yıldız Yapılarının Spitzer Araştırması $\left(S^{4} \mathrm{G}\right)$ örneklemesine ek olarak onun uzantısı 400 galaksiden oluşan örneklemeden orta-kırmızı öte renk seçim yöntemi ile seçilmiş 36 AGN kullanılmıştır. Kırmızı ötesi renk seçimi yöntemi, XMM-Newton ve Chandra verileri ile galaksilerin X-1şını özellikleri incelenerek test edilmiştir. X-1şını verilerini kullanarak, sıcak orta kırmızı ötesi çekirdek sergileyen galaksilerin (aday) aktif galaksiler olarak öne çıktığı gösterilmiştir. Orta-kırmızı ötesi seçilmiş AGN'lerin \%64'ü XMM-Newton ve Chandra verilerinde X-1şını enerjilerinde tespit edilmiştir. X-1şını tespitleri olmayan AGN renklerine sahip IRAC kaynakların büyük çoğunluğunun yüksek 1şıtmalı örtülmüş AGN'ler olduğu varsayılmıştır.

Anahtar Kelimeler: galaksiler: aktif - galaksiler: çekirdekler - kırmızı öte: galaksiler - X-ışınları: galaksiler

\section{Introduction}

Galaxies are known to contain supermassive black holes (e.g., Ferrarese and Merritt, 2000) in their centers, whose masses correlate with the masses of their bulges, indicating that the processes of forming bulges and supermassive black holes are intimately related (Magorrian

\footnotetext{
*Corresponding Author: tubaaaikiz@gmail.com
} 
et al., 1998; Tremaine et al., 2002). These supermassive black holes reside in all massive galaxies and growth and evolution of galaxies is closely linked to the growth and evolution of the supermassive black holes (e.g., Kauffmann and Haehnelt, 2000; Heckman, 2008). A small fraction of galaxies also has a very bright nucleus called an Active Galactic Nucleus (AGN), showing excess emission due to accretion of mass by the supermassive black hole that situates at the center of the galaxy. It is thought that AGN plays a dominant role during the formation of galaxies by creating large outflows that quench star formation in the galaxy (Kormendy and Ho, 2013). The nucleus of active and inactive galaxies are thus an ideal laboratory for studying the evolution and formation of galaxies. AGNs are among the most luminous objects in the universe and identifying these objects in all states of accretion and obscuration and accurately understanding their properties and structure is a key step to understand how galaxies evolve with cosmic time. Recent theoretical work suggests that feedback from AGN plays a significant role in establishing the present-day appearances of galaxies (e.g., Silk and Rees, 1998; Hopkins et al., 2008).

AGN can be defined in several ways at essentially all wavelengths (mid-infrared, X-rays, and radio detections and optical emission line diagnostics). Selection of AGN in the mid-infrared allows the exploration of strong AGN and quasars whose optical and soft X-ray emission is hidden by dust (e.g. Lacy et al., 2004, 2007, 2015; Stern et al., 2005; Martínez-Sansigre et al., 2005; Stern et al., 2012; Donley et al., 2012; Eisenhardt et al., 2012; İkiz et al., 2020). Highenergy radiation from the AGN is reprocessed by dust near the AGN and reradiated at midinfrared wavelengths. Luminous AGNs show a red mid-infrared power-law spectral energy distribution (SED), which is dominated by thermal emission from hot dust (Neugebauer et al., 1979; Elvis et al., 1994; Rieke and Lebofsky, 1981). Mid-infrared identification is sensitive to both obscured and unobscured AGNs, without requiring deep X-ray survey data. Emission at mid-infrared wavelengths can also possibly be used to detect Compton-thick AGNs that may be unnoticed by deep X-ray surveys (e.g. Ivison et al., 2004; Lacy et al., 2004; Stern et al., 2005; Alonso-Herrero et al., 2006; Polletta et al., 2006).

The presence of an extremely hot accretion disk in combination with a surrounding molecular torus, AGNs can be studied across the entire electromagnetic spectrum. The central region produces large amounts of X-rays which heat and ionize the surrounding gas and dust. Their X-ray continuum can be detected by X-ray satellites. Their emission may be weakened or blocked completely depending on the gas column density along the line of sight. The hard (2$10 \mathrm{keV}$ ) X-ray and mid-infrared wavebands provide powerful, complementary methods for identifying and studying AGN over a wide range of intrinsic obscuration (Eckart et al., 2018).

\section{Material and Methods}

Spitzer Space Telescope is a powerful tool for studying AGN demographics. In this study we used the Spitzer Survey of Stellar Structures in Galaxies ( $\mathrm{S}^{4} \mathrm{G}$; Sheth et al., 2010) AGNs that are detected by infrared excess method by İkiz et al., (2020). They showed that this method is one of the best ways of distinguishing AGNs from normal galaxies and an efficient way of finding AGNs in a large sample. The $S^{4} \mathrm{G}$ is a volume, magnitude, and size-limited survey of more than 2500 nearby galaxies using the IRAC Infrared Array Camera (Fazio et al., 2004) with deep imaging at 3.6 and $4.5 \mu \mathrm{m}$ on board the Spitzer Space Telescope which one of the Spitzer Legacy Programs of late type galaxies. This survey contains galaxies within $40 \mathrm{Mpc}$ $(\mathrm{v}<3000 \mathrm{~km} / \mathrm{s})$, away from the galactic plane $\left(|\mathrm{b}|>30^{\circ}\right)$, with extinction corrected B-band magnitude brighter than 15.5 and B-band diameter larger than 1' with distances determined from HI radial velocities. The $S^{4} G$ sample had been expanded with new $S^{4} G$ Extension 
observations for elliptical galaxies. Because galaxies need to contain HI many ellipticals are not included in the original $\mathrm{S}^{4} \mathrm{G}$ sample, and therefore the observations for the $\mathrm{S}^{4} \mathrm{G}$ Extension sample were taken. In İkiz et al., (2020) study, $2741 \mathrm{~S}^{4} \mathrm{G}$ sample galaxies are analyzed using mid-infrared excess method and 36 galaxies are detected candidate AGNs with searching counterparts in the Faint Images of the Radio Sky at Twenty-Centimeters (FIRST) and the National Radio Astronomical Observatory VLA Sky Survey (NVSS) survey catalogues. In this study $36 \mathrm{~S}^{4} \mathrm{G}$ AGNs are examined in XMM-Newton and Chandra survey catalogues to detect X-ray counterparts. All compact X-ray sources with a luminosity above $\approx 10^{42} \mathrm{erg}$ $\mathrm{s}^{-1}(2-10 \mathrm{keV})$ are considered to be AGN. Because of the broad wavelength coverage of the Xray band, there is a remarkable difference between the soft-band $(0.2-2 \mathrm{keV})$, the hard-band (2-6 keV) and the very hard-band (5-10 keV) surveys. The soft X-ray selection applicatively finds broad-line quasars and narrow line Seyfert galaxies (these objects tend to have a bright, soft spectral component, in addition to a flat, high energy, power-law spectrum). The hard Xray selection finds, in addition to classical Seyfert 1 galaxies and quasars, large numbers of objects with weak or absent optical emission lines and lacking non-thermal nuclei. X-ray surveys with XMM-Newton and Chandra at energies $<10 \mathrm{keV}$ are sensitive to all but the most heavily obscured AGN.

\section{Results and Discussion}

$\mathrm{S}^{4} \mathrm{G}$ survey infrared detected candidate AGNs are examined in the archives of X-ray surveys. $X M M-N e w t o n$ has a large field of view of $30^{\prime \prime}$ it provides a rich resource for serendipitous data thus providing a major resource, a deep, large-area sky survey. In the 3XMM-DR5 catalogue, the detection is considered as an extended source (Rosen et al., 2016). The Chandra Source Catalog (CSC) 1.1 release includes point and compact sources with observed spatial extents < 30" (Evans et al. 2010). In this study, AGN candidate counterparts are identified in 3XMM-DR5 and Chandra catalogues within 30". The 36 AGNs are listed in Table 2; well-known Seyfert galaxies NGC 1068, NGC 4051, and NGC 4151 are clearly detected.

$64 \%$ of $\mathrm{S}^{4} \mathrm{G}$ galaxies are detected in X-ray surveys as a hot-core (AGN candidate). Our detection statistics are not as high as optical spectroscopic surveys, but using the mid-infrared selection method with X-ray comparison is also effective to find AGN. In comparison with the study of İkiz et al., (2020) although the X-ray core detection is not as much as radio detection (88\%), it has enabled the counterparts of a large majority of AGNs. In addition, NGC 0625, NGC 7552 and NGC 7582 galaxies were detected in X-ray survey data, while they could not be detected in radio survey data.

Table 1. Fractions and numbers of hot- and cold-core galaxies detected in the two X-ray surveys

\begin{tabular}{|l|c|c|}
\hline Survey & Fraction & Hot-core $\left(\mathbf{S}^{\mathbf{4}} \mathbf{G}\right)$ \\
\hline X-ray & $63.9 \pm 8.0$ & $23 / 36$ \\
\hline XMM-Newton & $58.3 \pm 8.2$ & $21 / 36$ \\
\hline Chandra & $36.1 \pm 8.0$ & $13 / 36$ \\
\hline Survey & Fraction & Cold-core $\left(\mathbf{S}^{4} \mathbf{G}\right)$ \\
\hline X-ray & $14.9 \pm 0.7$ & $402 / 2705$ \\
\hline XMM-Newton & $10.8 \pm 0.6$ & $292 / 2705$ \\
\hline Chandra & $10.1 \pm 0.6$ & $227 / 2705$ \\
\hline
\end{tabular}


Notes. The hot-core galaxies are the Spitzer-detected AGN detected in the X-ray, while the cold-core galaxies are only detected in the X-ray and not as infrared-detected AGN.

Table 2. Detection of Spitzer selected AGNs in two X-ray surveys.

\begin{tabular}{|c|c|c|c|c|c|c|c|}
\hline Galaxy & $\begin{array}{c}\mathbf{R A} \\
(\mathrm{deg}) \\
(2)\end{array}$ & $\begin{array}{c}\text { DEC } \\
(\mathrm{deg}) \\
(3)\end{array}$ & $\begin{array}{l}\text { TT } \\
(4)\end{array}$ & $\begin{array}{l}\text { [3.6]-[4.5] } \\
\quad(\mathrm{mag}) \\
(5)\end{array}$ & $\begin{array}{c}\text { Error } \\
(\mathrm{mag}) \\
(6)\end{array}$ & $\begin{array}{c}\text { X-ray } \\
\text { (Detected/ } \\
\text { Non-detected) } \\
(7)\end{array}$ & $\begin{array}{c}\text { Survey } \\
(X M M-N e w t o n, \text { Chandra }) \\
(8)\end{array}$ \\
\hline ESO 409-015 & 1.38364 & -28.09991 & 5.4 & 0.800 & 0.413 & Non-detected & - \\
\hline NGC 0253 & 11.86515 & -31.42178 & -1.2 & 0.789 & 0.021 & Non-detected & - \\
\hline NGC 0520 & 21.14538 & 3.79159 & 1.3 & 0.522 & 0.043 & Detected & XMM-Newton,Chandra \\
\hline NGC 0625 & 23.76455 & -41.43722 & 9.0 & 0.688 & 0.137 & Detected & XMM-Newton,Chandra \\
\hline NGC 0660 & 25.75969 & 13.64581 & 1.3 & 0.568 & 0.026 & Detected & XMM-Newton,Chandra \\
\hline NGC 0814 & 32.65672 & -15.77344 & -1.7 & 0.744 & 0.108 & Non-detected & - \\
\hline PGC 009354 & 36.88651 & -10.16587 & 5.1 & 0.843 & 0.199 & Non-detected & - \\
\hline NGC 1068 & 40.66962 & -0.01331 & 3.0 & 0.797 & 0.017 & Detected & XMM-Newton,Chandra \\
\hline NGC 1365 & 53.40155 & -36.14039 & 3.2 & 0.679 & 0.020 & Detected & XMM-Newton \\
\hline IC 1953 & 53.42431 & -21.47868 & 6.2 & 0.989 & 0.150 & Non-detected & - \\
\hline NGC 1386 & 54.19239 & -35.99920 & -0.7 & 0.783 & 0.033 & Detected & XMM-Newton,Chandra \\
\hline NGC 3034 & 148.96800 & 69.67975 & 7.5 & 0.690 & 0.013 & Detected & XMM-Newton \\
\hline NGC 3094 & 150.35812 & 15.77011 & 1.1 & 0.950 & 0.028 & Detected & XMM-Newton \\
\hline NGC 3227 & 155.87740 & 19.86513 & 1.5 & 0.590 & 0.026 & Detected & XMM-Newton \\
\hline NGC 3516 & 166.69780 & 72.56850 & -2.0 & 0.519 & 0.035 & Detected & XMM-Newton \\
\hline UGC 06433 & 171.38258 & 38.06064 & 9.2 & 0.834 & 0.308 & Non-detected & - \\
\hline NGC 3729 & 173.45578 & 53.12555 & 1.2 & 0.704 & 0.067 & Non-detected & - \\
\hline NGC 4051 & 180.79007 & 44.53131 & 4.0 & 0.748 & 0.030 & Detected & XMM-Newton \\
\hline NGC 4151 & 182.63561 & 39.40578 & 2.0 & 0.988 & 0.029 & Detected & XMM-Newton,Chandra \\
\hline NGC 4293 & 185.30347 & 18.38261 & 0.3 & 0.635 & 0.048 & Non-detected & - \\
\hline NGC 4388 & 186.44490 & 12.66209 & 2.8 & 0.855 & 0.033 & Detected & XMM-Newton,Chandra \\
\hline NGC 4355 & 186.72764 & -0.87767 & 1.1 & 1.358 & 0.066 & Detected & Chandra \\
\hline NGC 4593 & 189.91437 & -5.34414 & 3.0 & 0.667 & 0.035 & Detected & XMM-Newton \\
\hline NGC 4628 & 190.60520 & -6.97103 & 2.9 & 0.812 & 0.045 & Non-detected & - \\
\hline ESO 443-042 & 195.87400 & -29.82870 & 3.0 & 0.597 & 0.091 & Non-detected & - \\
\hline NGC 4968 & 196.77420 & -23.67690 & -2.0 & 0.744 & 0.045 & Detected & XMM-Newton \\
\hline NGC 5253 & 204.98315 & -31.64006 & 8.9 & 1.298 & 0.027 & Detected & XMM-Newton \\
\hline NGC 5347 & 208.32421 & 33.49085 & 2.0 & 0.890 & 0.044 & Detected & Chandra \\
\hline NGC 5427 & 210.85854 & -6.03075 & 5.0 & 0.506 & 0.082 & Non-detected & - \\
\hline NGC 5506 & 213.31209 & -3.20757 & 1.2 & 1.059 & 0.029 & Detected & XMM-Newton,Chandra \\
\hline NGC 5861 & 227.31709 & -11.32171 & 5.0 & 1.601 & 0.084 & Non-detected & - \\
\hline NGC 7314 & 338.94252 & -26.05043 & 4.0 & 0.712 & 0.047 & Detected & XMM-Newton,Chandra \\
\hline NGC 7378 & 341.94867 & -11.81664 & 2.2 & 0.803 & 0.072 & Non-detected & - \\
\hline NGC 7479 & 346.23590 & 12.32293 & 4.3 & 1.150 & 0.041 & Detected & XMM-Newton \\
\hline NGC 7552 & 349.04494 & -42.58496 & 2.4 & 0.511 & 0.028 & Detected & XMM-Newton,Chandra \\
\hline NGC 7582 & 349.59837 & -42.37034 & 2.1 & 0.890 & 0.026 & Detected & XMM-Newton,Chandra \\
\hline
\end{tabular}

Notes. Columns are: (1) Galaxy name; (2) Right ascension (J2000); (3) Declination (J2000); (4) Numerical morphological type; (5) [3.6]-[4.5] color (İkiz et al. 2020); (6) [3.6]-[4.5] color error (İkiz et al. 2020); (7) X-ray (Detected/Non-detected); (8) Survey. Column (7) indicates whether AGN detected or non-detected in both surveys. Column (8) refers to whether AGN are detected in the archives of XMM-Newton and Chandra within $30^{\prime \prime}$ radius, respectively. (-) refers to the lack of data in both surveys.

Table 2 shows that about 36\% (13 out of 36) of the infrared-detected candidate AGN have never before been detected as AGN in X-ray surveys. The two individual X-ray surveys are illustrated in the histograms of Figure 1. This figure shows that XMM-Newton survey detection is more sensitive than Chandra to find hot-core candidate AGN. It was determined that ESO 409-015, NGC 0253, UGC 06433 and NGC 7378 galaxies are defined as nondetected AGNs in both X-ray surveys mentioned in this study and also radio surveys refers in İkiz et al., (2020) study. 


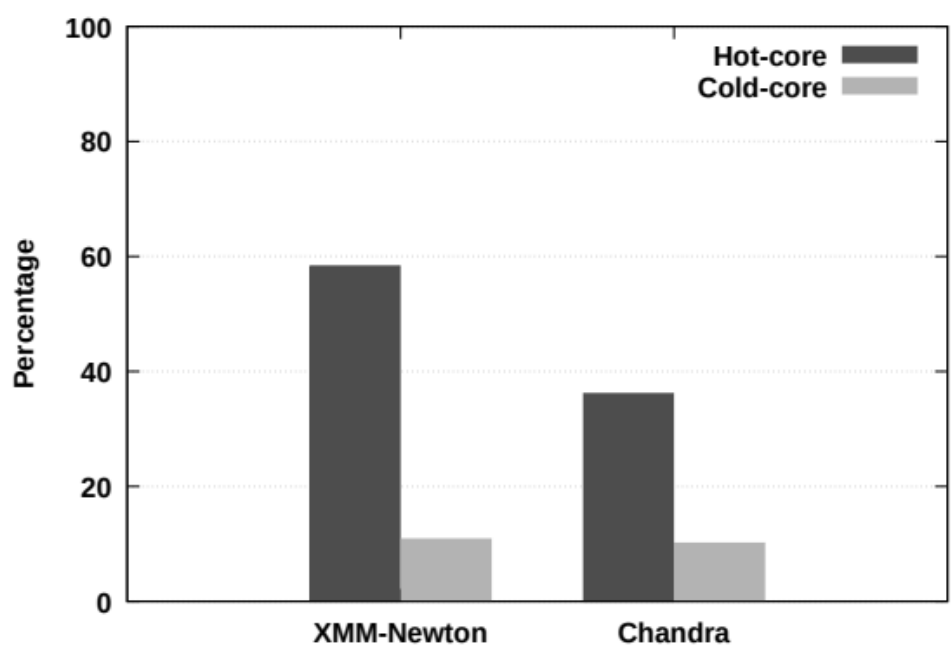

Figure 1. The fraction of Spitzer detected candidate AGN also detected in other surveys for XMM-Newton and Chandra (in dark colors in the histogram). Light colors in the histogram refer to the candidate non-AGN.

\section{Conclusion}

$\mathrm{X}$-ray selection is one of the ways of finding active galaxies. It has been known over 40 years that the great majority of high latitude, point-like "hard" X-ray sources are AGN. Like radio emission, luminous, compact X-ray emission is almost a certain identifier of the existence of an AGN and does not need "confirmation" by data in other wavelength bands (Mushotzky, 2004). X-ray emission originates from very close to the central black hole, often showing large amplitude, rapid variability, and being characterized by a non-thermal spectrum (Mushotzky et al., 1993). Thus the X-ray properties are directly connected to the black hole nature of the AGN and are not due to reprocessing of the radiation.

XMM-Newton and Chandra are very sensitive to the presence of AGN. Sources in the luminosity range $10^{42}-10^{46} \mathrm{erg} \mathrm{s}^{-1}$ can be detected out to $\mathrm{z} \sim 3$, independent of the nature of the host galaxy (Steffen et al., 2003). In the low redshift universe, there are no galaxies with an aggregate (non-AGN) luminosity exceeding this level. Thus, even without detailed X-ray spectra or imaging, the identification of the nature of the source is clear. As such, IRAC selection is a powerful technique not only for detecting heavily obscured AGNs missed in the $\mathrm{X}$-ray, but also selecting luminous obscured and unobscured AGNs when deep X-ray data are absent. These results show that $2-10 \mathrm{keV}$ X-ray surveys are efficient at finding candidate AGN sources missed by mid-infrared selection techniques. Many of the X-ray undetected Spitzer AGN are heavily obscured, resulting in faint optical counterparts and X-ray nondetection.

\section{References}

Alonso-Herrero, A. et al. 2006. Infrared Power-Law Galaxies in The Chandra Deep FieldSouth: Active Galactic Nuclei and Ultraluminous Infrared Galaxies, The Astrophysical Journal, 640:167-184.

Della Ceca R., et al., 2008. The Cosmological Properties of AGN in The XMM-Newton Hard Bright Survey, Astronomy \& Astrophysics, 487, 119-130. 
Donley, J. L., Koekemoer, A. M., Brusa, M., et al. 2012. Identifying Luminous Active Galactic Nuclei in Deep Surveys: Revised IRAC Selection Criteria, The Astrophysical Journal, 748:142.

Eckart, A., M. Zajacek, M. Parsa, et al. 2018. The Multifrequency Behavior of Sagittarius A*, Proceedings of Science, ArXiv:1806.00284.

Elvis M. et al., 1994. Atlas of Quasar Energy Distributions, The Astrophysical Journal Supplement Series, 95:1-68.

Evans I. N., et al., 2010. The Chandra Source Catalog, The Astrophysical Journal Supplement Series, 189:37-82.

Fazio, G. G., Hora, J. L., Allen, L. E., et al. 2004. The Infrared Array Camera (IRAC) For The Spitzer Space Telescope, The Astrophysical Journal Supplement Series, 154:10-17.

Ferrarese, L. \& Merritt, D. 2000. A Fundamental Relation Between Supermassive Black Holes and Their Host Galaxies, The Astrophysical Journal, 539: L9-L12.

Heckman, T. M. 2008. The Escape Fraction of Ionizing Photons from High Redshift Galaxies from Data-constrained Reionization Models, Monthly Notices of the Royal Astronomical Society, ArXiv:1207.3803v2.

Hopkins, P. F., Hernquist, L., Cox, T. J., and Kereš, D. 2008. A Cosmological Framework for The Co-Evolution of Quasars, Supermassive Black Holes, And Elliptical Galaxies. I. Galaxy Mergers and Quasar Activity, The Astrophysical Journal Supplement Series, 175:356-389.

İkiz, T., Peletier, R.F., Barthel, P., et al. 2020. Infrared-detected AGNs in The Local Universe, Astronomy \& Astrophysics, 640, A68.

Ivison, R. J., et al. 2004. Spitzer Observations of Mambo Galaxies: Weeding Out Active Nuclei in Starbursting Protoellipticals, The Astrophysical Journal Supplement Series, 154:124-12.

Kauffmann G. Haehnelt M., 2000. A Unified Model for The Evolution of Galaxies and Quasars Monthly Notices of the Royal Astronomical Society, 311, 576-588.

Kormendy, J. \& Ho, L. C. 2013. Coevolution (Or Not) Of Supermassive Black Holes and Host Galaxies: Supplemental Material, ArXiv:1308.6483.

Lacy, M., Petric, A. O., Sajina, A., et al. 2007. Optical Spectroscopy and X-Ray Detections of a Sample of Quasars and Active Galactic Nuclei Selected in The Mid-Infrared from Two Spitzer Space Telescope Wide-Area Surveys, The Astronomical Journal, 133:186-205.

Lacy, M., Ridgway, S. E., Sajina, A., et al. 2015. The Spitzer Mid-Infrared AGN Survey. Ii. The Demographics and Cosmic Evolution of The AGN Population, The Astrophysical Journal, 802:102.

Lacy, M., Storrie-Lombardi, L. J., Sajina, A., et al. 2004. Obscured and Unobscured Active Galactic Nuclei in The Spitzer Space Telescope First Look Survey, The Astrophysical Journal Supplement Series, 154:166-169. 
Magorrian, J., Tremaine, S., Richstone, D., et al. 1998. The Demography of Massive Dark Objects in Galaxy Centers, The Astronomical Journal, 115:2285-2305.

Martínez-Sansigre, A., Rawlings, S., Lacy, M., et al. 2005. The Obscuration by Dust of Most of the Growth of Supermassive Black Holes, Nature, 436: 666-669.

Mushotzky, R. 2004, in Astrophysics and Space Science Library, Vol. 308, Supermassive Black Holes in The Distant Universe, ed. A. J. Barger, 53.

Mushotzky, R. F., Done, C., \& Pounds, K. 1993. X-ray Spectra and Time Variability of Active Galactic Nuclei, The Annual Review of Astronomy and Astrophysics, 31:717-717.

Neugebauer G., Oke J. B., Becklin E. E., Mathews K., 1979. Absolute Spectral Energy Distribution of Quasi-stellar Objects Ffom 0.3 To 10 Microns, The Astrophysical Journal, 230: 79-94.

Polletta, M.D.C., et al. 2006. Chandra and Spitzer Unveil Heavily Obscured Quasars in The Chandra/SWIRE Survey, The Astrophysical Journal, 642:673-693.

Rieke, G. H., \& Lebofsky, M. J. 1981. Spectral Components of NGC 4151, The Astrophysical Journal, 250:87-97.

Rosen, S. R., Webb, N. A., Watson, M. G., et al. 2016. The XMM-Newton Serendipitous Survey: VII. The Third XMM-Newton Serendipitous Source Catalogue, Astronomy and Astrophysics, 590, A1.

Sheth, K., Regan, M., Hinz, J. L., et al. 2010. The Spitzer Survey of Stellar Structure in Galaxies (S4G), Publications of The Astronomical Society of the Pacific, 122:1397-1414.

Silk J., Rees M. J., 1998. Quasars and Galaxy formation, Astronomy and Astrophysics, 331, L1-L4.

Steffen A. T., Barger A. J., Cowie L. L., Mushotzky R. F., Yang Y., 2003a. The Changing Active Galactic Nucleus Population, The Astrophysical Journal, 596: L23-L26.

Stern, D., Assef, R. J., Benford, D. J., et al. 2012. Mid-Infrared Selection of Active Galactic Nuclei with The Wide-Field Infrared Survey Explorer. I. Characterizing Wise-Selected Active Galactic Nuclei in Cosmos, The Astrophysical Journal, 753:30.

Stern, D., Eisenhardt, P., Gorjian, V., et al. 2005. Mid-Infrared Selection of Active Galaxies, The Astrophysical Journal, 631:163-168.

Tremaine, S., Gebhardt, K., Bender, R., et al. 2002. The Slope of the Black Hole Mass Versus Velocity Dispersion Correlation, The Astrophysical Journal, 574:740-753.

3XMM-DR5 Catalogue.

http://xmmssc.irap.omp.eu/Catalogue/3XMM-DR5/3XMM_DR5.html

The Chandra Source Catalogue Release 1.1. https://cxc.cfa.harvard.edu/csc1/ 